\title{
REAL-TIME DROWSINESS DIAGNOSTIC SYSTEM USING OPENCV ALGORITHM
}

\author{
Deeksha Phayde ${ }^{1}$, Pratima Shanbhag ${ }^{1}$, Subramanya G. Bhagwath ${ }^{2}$ \\ ${ }^{1}$ UG Students, ${ }^{2}$ Assistant Professor ,Dept. of Computer Science Engineering, \\ Anjuman Institute of Technology and Management, Bhatkal, Karnataka, India
}

\begin{abstract}
Drowsiness or fatigue is a major cause of road accidents and has a significant impact on road safety. There are many fatal accidents that can be avoided if drowsy drivers are warned early. There are a variety of sleep detection systems available that monitor drivers' drowsiness while driving and alert drivers if they are not focused on driving. Appropriate features can be extracted from facial expressions such as yawning, eye closing, and head movement to determine the level of sleepiness. The biological condition of the driver's body, as well as the behavior of the vehicle, are analyzed to determine if the driver is drowsy. It presents a comprehensive analysis of the available mechanisms for the driver's drowsiness and presents a detailed analysis of the most commonly used classification strategies in this regard. We divide existing strategies into three categories: behaviors, physical, and strategies based on life parameters. Second, the supervised learning methods used for sleep apnea are being reviewed. Third, the pros and cons and comparative research of different approaches are discussed. In addition, the research frameworks are detailed in the diagrams for better understanding. Because of the dangers posed by road fatigue, researchers have developed various mechanisms to detect driver drowsiness and each procedure has its own benefits and limitations. In order to make an important review of Drowsiness Detection Techniques (DDT) and appropriate classification methods, we created a search engine unit to gather relevant information. We keep our search focused on publishing reputable journals and conferences. We have developed a multi-stage selection process and testing process.
\end{abstract}

Keywords: Drowsiness detection System, OpenCV

\section{INTRODUCTION}

With the increase in the number of vehicles worldwide, road accidents have become one of the leading causes of death. According to a report by the World Health Organization (WHO), road accidents are one of the ten leading causes of death in 2015. As we know, the driver, as the root of the road transport system, is the most important factor affecting road traffic safety[1,2].

According to a National Sleep Foundation record, about $32 \%$ of drivers have at least one driver experience accident per month. There are about 100000 accidents caused by drowsiness and about $25 \%$ of road accidents involve drowsiness every year. Drowsiness driving refers to the behavior of driving skills that decreases in a direct way, due to the imbalance in physical activity after continuous driving for a long period of time. It may affect driving behavior and be a major cause that threat to drivers' safety and other road users' live.[ 3].

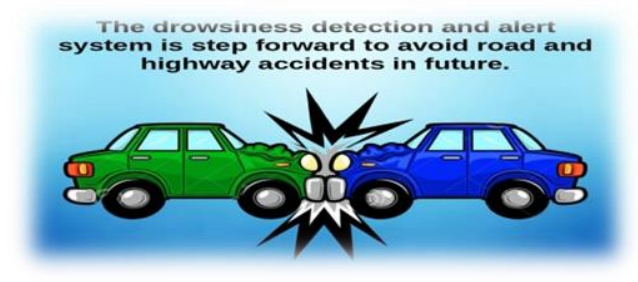

Fig.1.1

Drowsiness or fatigue is one of the most important factors that endanger road safety and cause serious injury, death and economic loss. Increased drowsiness impairs driving performance. Lack of alertness, caused by the unconscious transition from waking to sleep, leads to several serious road accidents.

However, this route cannot detect whether the driver is tired or not in real-time. With the advent of information technology, the sleep drill system has become another way to solve the problem. Therefore, research on the intelligent detection of drowsiness has important practical implications. 


\section{International Journal of Trendy Research in Engineering and Technology \\ Volume 6 Issue 2 April 2022 \\ ISSN NO 2582-0958}

In recent years, due to less aggressive and low cost, methods of driving drowsiness based on driver behavior have become the subject of research. However, the effectiveness of algorithms may limit the ability to detect facial expressions and assess fatigue in a complex and flexible environment.

\subsection{Problem Definition}

According to a study, $32 \%$ of road accidents are caused by the driver's drowsiness. This becomes a major problem not only for the driver but also for other people who use the road. What's more, when things get really bad, it could be a danger in a crowded place or a school place. Thus, this Drowsiness Driver diagnostic program is a safe system that warns the driver whenever he feels drowsy. The driver's eye movement is monitored live and whenever the driver feels asleep or closes the eye for more than 2 seconds then with the help of an alarm. Thus, preventing any accidents from occurring [4].

\subsection{Existing System}

Drowsiness can be detected with the help of photos or realtime video taken from a camera mounted in front of the driver's face. This video is converted to a frame. OpenCVclassifier uploaded in the system. Each frame is compared with the previously defined features of OpenCV classifiers. When features are matched to the face it is found and the rectangle is drawn on the face. By using the extraction feature, we measure the eye area. Compared to the classifier OpenCV, eyes are detected and rectangles are drawn around the left and right eyes.

Choudhary et al[5] have proposed an approach using OpenCV-based cascade classifiers. They have used the Haar-based cascade classifier to detect eye movement and OpenCV to identify the driver's state effectively Additionally their system can work in adverse conditions, such as varying lighting conditions, use of spectacles, and the presence of a beard on the person's face. Their system can also classify drowsiness in such extreme conditions as well accurately.

Due to differences in facial features, the OpenCV feature works best in real-time facial detection. These can be calculated according to the difference in the total number of pixels within a rectangular area and during the process. The Adaboost algorithm will allow all face samples and will discard non-surface image samples [1].

\subsection{Proposed System}

\subsubsection{Face and Eye Detection by OpenCV Algorithms:}

In this paper, a new approach to sensitive face detection is provided based on analogic cellular neural network (OpenCV) algorithms. The proposed OpenCV algorithms detect and help to detect people's faces and drowsiness effectively which is the cause of many accidents related to car crashes. Driver fatigue detection is a part of the previously used methods. The algorithm begins with the acquisition of data about heads in colored images using color distortion and the structure of the human face and background[ 3].

Depending on the distance and location of the reference points, all faces should be adjusted to the same size and shape. To get used to it, the eyes serve as a point indicator. Another OpenCV algorithm catches the eyes in any gray image by searching for key features of the eyes and eye foundations. Tests conducted on a standard website show that the algorithm works very fast and reliably

Fig.1.3.1 describes the proposed system, in the first step an image is captured by a webcam for processing. Driver photos are taken from the installed camera. It will be forwarded to pre-processing which prepares the image for further processing by the system. If no face is found another frame will be searched. If a face is found, then the interesting region is marked inside the face. This is a region of interest that consists of eyes.

Defining a region of interest greatly reduces the calculation requirements of the system. After that, the eyes are found in the area of interest. If the eye is detected, if there is no blink means the driver is not feeling drowsy. The blink counter is set to "20" if the system detects the blinking of eyes. When the eyes are closed in a certain frame, the blink counter is reduced and blinks are detected. If the eyes are closed for more than 4 or 5 frames, then it is found that the driver feels drowsy. Therefore, drowsiness is detected and the alarm goes on. After this, the whole process is repeated as long as the driver is driving the car until he/she gets back to the active state. 


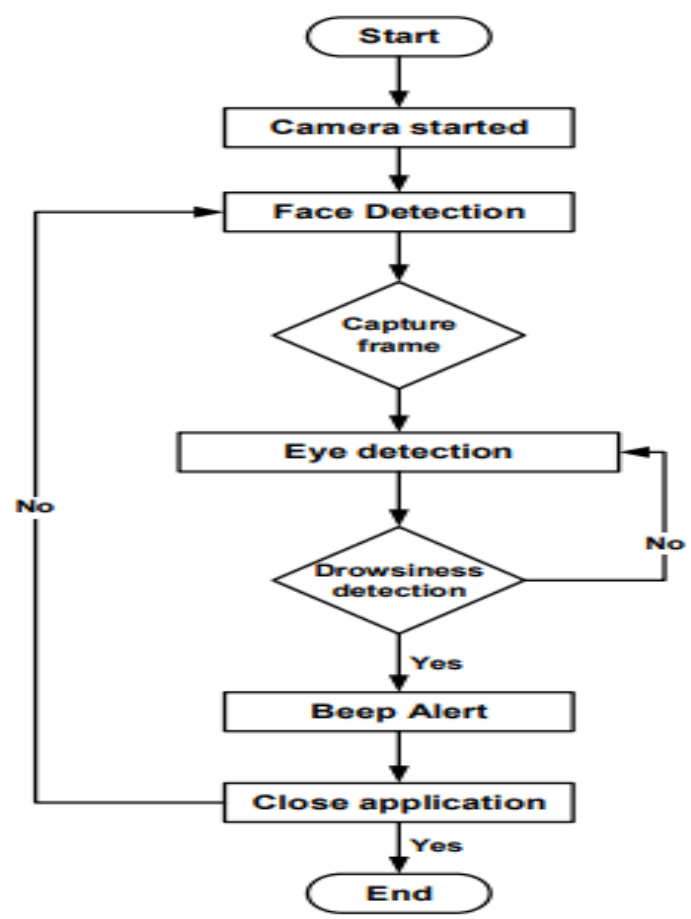

Fig.1.3.1 Flowchart of Driver Drowsiness Detection System

\subsection{Objectives}

$>$ Our proposed approach is to design and develop a low-cost, embedded-based system.

$>$ The main purpose is to prepare a prototype for the driver's drowsiness system that alerts the driver when there is drowsiness.

$>$ Many of the methods available to get detect drowsiness are based on face or eye to determine the degree of disturbance of the driver.

$>$ This method involves the condition of both eye states to detect the drowsiness of the driver.

$>$ Achieving the use of a computer model for driver identification and analysis of driver status.

$>$ The detection system analyzes the frame by frame and determines whether the driver's eyes are open or closed, depending on the Eye aspect ratio then the system alerts the sound.

\subsection{Salient Features}

$>$ Driver fatigue and their requirement are part of the previously used methods.
The algorithm starts with the acquisition of data regarding faces in colored images using the deviation of background and structure of the human face.

$>$ Other OpenCV algorithms find the eyes in any gray image by searching for features of the eyes and eye basis.

$>$ The algorithm requires both good and bad images to train the detector.

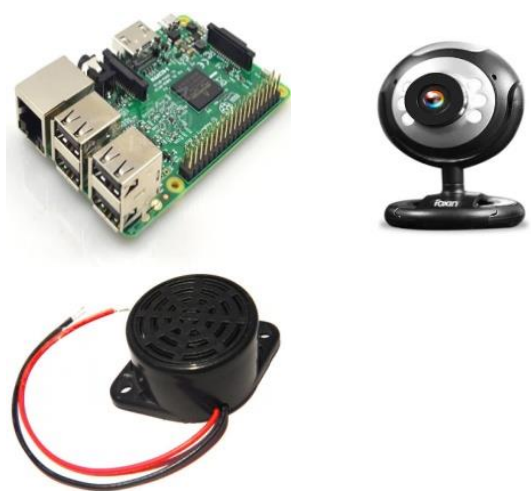

Fig. 1.5.1Raspberry pi, Web cam, Buzzer

\subsection{Advantages}

$>$ The drowsiness detection system will detect a problem before any problem accord and inform the driver and other passengers by raising an alarm.

$>$ In this, OpenCV-based machine learning techniques are used for the automatic detection of drowsiness.

\section{LITERATURE SURVEY}

\section{A. Visual Analysis of Eye State and Head Pose for Driver Alertness Monitoring}

They presented a visual analysis of eye state and head pose (HP) for continuous monitoring and alertness of a driver. Most existing approaches to visual detection are by driving patterns which rely either on eye closure or headnodding angles to determine the driver's drowsiness or distraction level. The proposed scheme uses visual features such as eye index (ei), pupil activity (pa), and hp to extract critical information on the alertness of a vehicle driver. A support vector machine (SVM) classifies a sequence of video segments into drowsy or non-drowsy driving events. The experimental results showed that the proposed scheme offers high classification accuracy with acceptably of low 


\section{International Journal of Trendy Research in Engineering and Technology \\ Volume 6 Issue 2 April 2022 \\ ISSN NO 2582-0958}

errors and false alarms for people of various ethnicity in real road driving conditions.

\section{B. Driver Monitoring Based on Low-Cost 3-D Sensor}

They proposed a solution for driver monitoring and event detection based on 3-D sensors information from camera. The system combines 2-D and 3-D techniques to provide head pose estimation and regions-of-interest identification. Based on the captured cloud of 3-D points from the sensor and analyzing the 2-D projection, the points corresponding to the head are determined and extracted for further analysis. Later, head pose estimation with three degrees of freedom (Euler angles) is estimated based on the iterative closest points algorithm. Finally, relevant regions of the face are identified and used for further analysis, event detection, and behavior analysis. The resulting application is a driver monitoring system based on low-cost 3D sensors. It represents an interesting tool for human research studies, allowing the automatic study of specific factors and the detection of special events related to the driver drowsiness, inattention, or head pose.

\section{A Real-Time Driving Drowsiness Detection}

\section{Algorithm with individual differences consideration[3]}

The proposed algorithm is to detect drowsiness of the driver, which considers the individual differences of the drivers. It consists of an off-line training module and online monitoring module. Firstly, they designed a Deep Cascaded Convolutional Neural Network (DCCNN) to detect the face region from live video. Based on this, the eyes landmarks are obtained by the application of Dlib toolkit. A new parameter, Eyes Aspect Ratio (EAR), which is calculated by the coordinates of landmarks, is introduced to identify the state of eyes (open or closed).

\section{Camera-based Drowsiness Reference for Driver State}

\section{Classification under Real Driving Conditions}

They suggested that the driver's eye movements could be detected by sleepiness under the test conditions. The latest eye-tracking based on measures of eye status for fatigue is being tested. These steps are calculated mathematically and categorically based on a large 90-hour database of real road driving. The results show that sleep detection works well for some drivers as long as blink vision is effective. Despite the proposed improvements, however, there are still problems with poor lighting conditions and with people wearing glasses. In short, camera-based sleep measurements provide an important contribution to the sleep indicator, but they are not reliable enough to be the only indicator.

\section{E. Real-time monitoring of driver drowsiness on mobile} platforms using 3D neural networks

New technologies have the potential to further reduce the number of injuries and deaths of road users by alerting drivers about sleep disorders. It is reported that drowsiness warnings lead drivers to look at the road faster In addition, Another report indicates

that the driver's response times from the departure lane will be better when the warning was given earlier.

\section{TOOLS AND TECHNOLOGY USED}

\subsection{Tools and image processing libraries}

Following optimized tools and image processing libraries are used for the implementation of the presented algorithm.

\section{OpenCV(Open- source Computer Vision):}

It has a wide range of modules that can help us with lot of computer vision problems. But perhaps the most useful part of OpenCV is its architecture and memory management. It provides with a framework in which we can work with images and videos, using OpenCV's algorithms, without worrying about allocating and reallocating memory for images. OpenCV libraries and functions are highly optimized and can be used for real-time image and video processing.

DLib:

It is a modern $\mathrm{C}++$ toolkit containing machine learning algorithms and tools for creating complex software in $\mathrm{C}++$ to solve real-world problems. It is used in both industry and academia in a wide range of domains including robotics, embedded devices, mobile phones, and large high-performance computing environments. Open Source Dlib library is used for the implementation of $\mathrm{CNN}$ (convolutional neural networks).

\section{Python:}

Python is an object-oriented programming language created by Guido Rossum in 1989. It is ideally designed for rapid prototyping of complex applications. It has interfaces to many OS system calls and libraries and is extensible to $\mathrm{C}$ or $\mathrm{C}++$. Many large companies use the Python programming language including NASA, Google, 


\section{International Journal of Trendy Research in Engineering and Technology \\ Volume 6 Issue 2 April 2022 \\ ISSN NO 2582-0958}

YouTube, etc. Python is widely used in Artificial Intelligence, Natural Language Generation, Neural Networks and other advanced fields. Python had deep focus on code readability. Python language is used due to the cross platform compatibility as main coding language for algorithm. OpenCV and Dlib libraries are integrated in Python interpreters for using readymade optimized functions.

\subsection{Technology Used}

\subsubsection{Machine Learning:}

Machine Learning is the kind of programming that gives computers the capability to automatically learn from data without being explicitly programmed. These programs change their behavior by learning from data. Python is clearly one of the best languages for machine learning. Python does contain special libraries for machine learning namely pandas, NumPy for linear algebra, and the kernel methods of machine learning. The language is to use when working with machine learning algorithms and has easy syntax relatively.

\subsubsection{OpenCV:}

It has a wide range of modules that can help us with lot of computer vision problems. But perhaps the most useful part of OpenCV is its architecture and memory management. It provides a framework in which we can work with images and videos, using OpenCV's algorithms, without worrying about allocating and reallocating memory for images. OpenCV libraries and functions are highly optimized and can be used for real-time image and video processing.

\section{REQUIREMENT ANALYSIS}

\subsection{Functional Requirements}

The requirement is defined as one or more product components, throughout the system. Volume or component is also shown as a category lead and its products, given the many data source. A useful requirement may be identified by special offers and footnotes or data management as well as the willingness of other utilities that define the intended purpose of a particular structure where the requirements apply to situations.[4]

$>$ Recording the driver's steering behavior when the movement begins.

$>$ Then recognizes changes over the long trip.

$>$ Determines the driver's level of fatigue.

\subsection{Non-Functional Requirements}

$>$ Image processing is done using the captured video.

$>$ Image is stored in a library called OpenCV.

$>$ Stored image undergo various algorithm and detects if the driver is fatigue.

\subsection{Hardware Requirements}

$>$ Personal PC or Laptop

$>$ Wireless Connection or connecting cable

$>$ The processoror of Intel or high level

\subsection{Software Requirements}

$>$ Windows 7 or more

$>$ Internet connection

$>$ OpenCV

$>\quad$ Python 2.7 or 3.9 version

\section{DESIGN}

The driver's face is constantly monitored using a camera. The first step is to detect the face using a series of frames taken by cameras. The eye area is then detected and the retina of the eye is examined continuously. The captured image is sent to the Raspberry Pi board for processing. The raspberry Pi converts a captured image into a digital signal using OpenCV. The digital signal is transmitted from the sender to the receiver. Both transmitter and receiver are paired. The signal is then transferred to LPC2148, a microcontroller [3,5].

When the signal exceeds the 2-second limit, the alarm goes on and the car speed drops automatically. An improved drowsiness detection system can detect drowsiness more quickly. During monitoring, the system is able to determine whether the eyes are open or closed. By using a drowsy detection system, driver safety can also be achieved in normal vehicles.

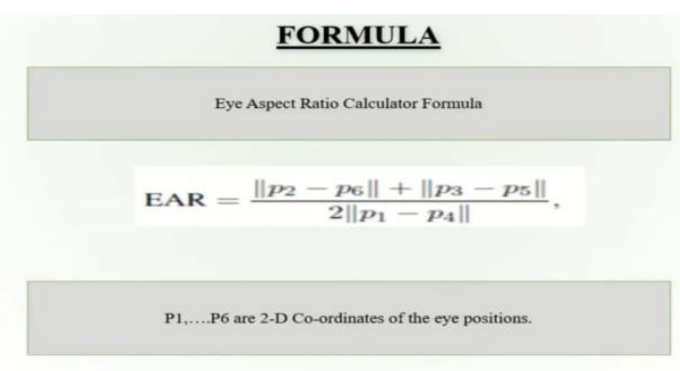

Fig5.1 Eye-Aspect Ratio formula 
This project is designed to achieve certain goals which are:

$>$ Drowsiness detection is a car safety technology that helps save the life of the driver by preventing accidents when the driver starts feeling drowsy.

$>$ The main purpose is to design a driver's fatigue detection system to detect drowsiness continuously and monitor the retina of the eye [3].

$>$ The system works to alert the driver of drowsiness using a buzzer or alarm.

$>$ The speed of the car can be reduced.

$>$ Traffic control can be maintained by reducing risks.

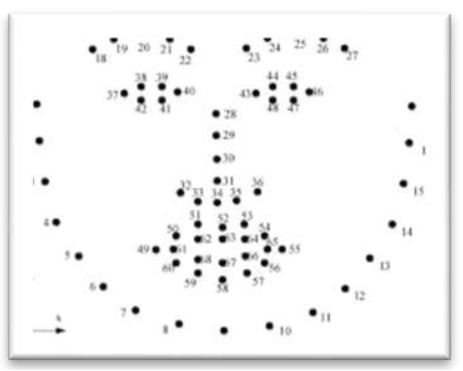

Fig. 5.2 Creating a Region of Interest (ROI) using Facial Co-ordinates

\subsection{System Architecture}

\subsubsection{Data Flow Diagram}

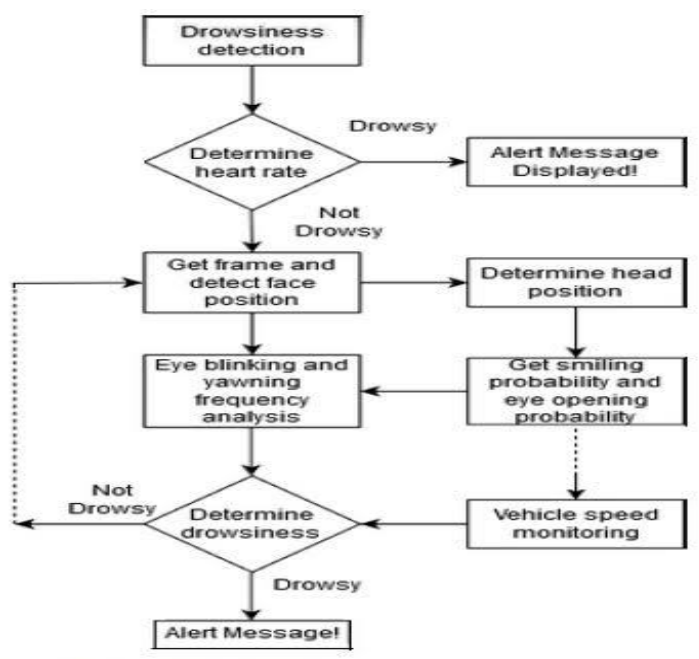

Fig.5.1.1 Data Flow Diagram for Drowsiness Detection System
Drowsiness Detection System: The image is captured and even processed using various algorithms to detect whether the driver is fatigued or not.

$>$ Driver: The person who is driving the vehicle.

$>$ Camera: Captures the video of the driver when the vehicle is in motion and sends the captured video to the storage unit.

> Facial Landmark Detection: Detects the driver's facial changes and reports them.

\section{CONCLUSION}

$>$ This program can be used to reduce the number of road accidents that occur most.

$>$ This can save many lives, which is the main motivation for this program.

$>$ Therefore, this program does not require any sophisticated system in order to be effective and can be used for road safety in the future.

\section{REFERENCES}

[1] Feng You, Xiaolong Li, Yunbo Gong, Haiwei Wang, And Hongyi Li, "Real-Time Driving Drowsiness Algorithm For Individual Differences Consideration", Ieee- Access, Special Section On Artificial Intelligence (Ai)-Empowered Intelligent Transportation Systems, 2019,7 Pp 179396179408 Doi: 10,2019. 10.1109/Access. 2019.

[2] Gaurav Kanojia, Vijay Kanojiya, Glenn Mendonza 'Detection Of Drowsiness And Distraction Of Drivers Using Cnn' Int. J. Trendy Res. Eng.(IJTRET) 4(7) 2020, p23-26

[3] Jasper S. Wijnands, Jason Thompson, Kerry A. Nice, Gideon D. P. A. Aschwanden, Mark Stevenson, "RealTime Monitoring Of Driver Drowsiness On Mobile Platforms Using 3d Neural Networks", Neural Comput \& Applic 32, 9731-9743 (2020)

[4] Ralph Oyini Mbouna And Seong G. Kong, "Visual Analysis Of Eye State And Head Pose For Driver Alertness Monitoring",Ieee Transactions On Intelligent Transportation Systems (2013)14(3):14621469.Doi: 10.1109/Tits.2013.2262098 
[5] Y. Choudhary, A. Aggarwal and A. Agarwal, "Detecting Drivers' Drowsiness using Haar Cascade Classifier," 2022 9th International Conference on Computing for Sustainable Global Development (INDIACom), 2022, pp. 318-322,

DOI: 10.23919/INDIACom54597.2022.9763192

\section{BIOGRAPHICS}

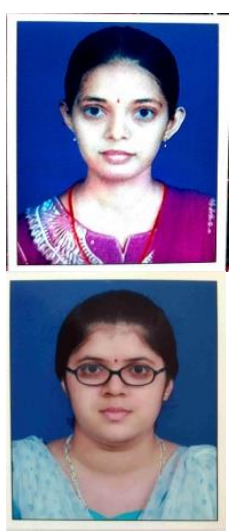

Deeksha Phayde

Final Year B.E Student

Department of Computer Science

Engineering , AITM, Bhatkal

Karnataka, Bhatkal

Pratima Shanbhag

Final Year B.E Student

Department of Computer Science

Engineering ,AITM, Bhatkal

Karnataka, Bhatkal 